\title{
EFECTO DEL RECTIFICADO Y DESGASTE DE CUCHILLOS EN LA CALIDAD SUPERFICIAL DE MOLDURAS DE PINO RADIATA
}

\section{JOINTING KNIVES AND TOOL WEAR EFFECT IN THE SURFACE QUALITY OF RADIATA PINE MOLDINGS}

\author{
Alfredo Aguilera ${ }^{1 *}$, Christopher Fernández ${ }^{1}$
}

\begin{abstract}
RESUMEN
Se estudia el efecto que ejerce el talón de rectificado de cuchillos en acero rápido sobre la calidad superficial de molduras sólidas de pino radiata. Se analizan los defectos fibra encontrada (grano velloso o fuzzy grain) y fibra astillada (grano arrancado o torn grain) en molduras de perfil plano que se generan en el proceso de moldurado. Se evaluó la relación talón de rectificado - calidad superficial, a través de dos procedimientos, en el primero se realiza el rectificado en forma sistemática, y en el segundo sólo una vez, al inicio del proceso, para en ambos casos cuantificar los defectos generados en las piezas. Los resultados indican que la aparición de fibra encontrada y fibra astillada se pueden relacionar con la distancia de corte y con el estado del talón de rectificado, asimismo, la calidad del material de corte y las condiciones de operación deben tenerse en cuenta ya que de estos factores depende el rendimiento del proceso.
\end{abstract}

Palabras claves: Fibra encontrada, fibra astillada, rectificado, pino radiata.

\begin{abstract}
The effect exerted by the land of jointing of high speed steel knives is studied on the surface quality of radiata pine solid moldings. Fuzzy grain and torn grain are analyzed on flat molding profile. To evaluate these defects, the relationship land of jointing - surface quality was assessed through two procedures, on the first the jointing was done systematically, and on the second only once, early in the process. The results indicate that fuzzy grain and torn grain are related to cutting distance and jointing process, the quality of the cutting material and operating conditions must be taken into account because of these factors depends the process performance as well.
\end{abstract}

Keywords: Fuzzy grain, torn grain, jointing, radiata pine.

\section{INTRODUCCIÓN}

El procedimiento de rectificado (jointing) de cuchillos tanto en máquinas cepilladoras como moldureras es una práctica común en la industria maderera, teniendo este procedimiento objetivos muy concretos: por una parte cuando los cabezales porta-herramientas poseen una cantidad importante de cuchillos, el montaje se hace cada vez más difícil e impreciso, por ello se hace necesario igualar la proyección de éstos, logrando un círculo de corte con tolerancias mínimas, e igualando además cualquier imperfección dentro de cada cuchillo. Además, debe considerarse que el rectificado

\footnotetext{
${ }^{1}$ Instituto de Tecnología de Productos Forestales, Facultad de Ciencias Forestales y de Recursos Naturales, Universidad Austral de Chile, Casilla 567, Valdivia. Chile. 
sólo es aplicable cuando el cabezal porta-herramientas posee un montaje de tipo hidro-centrado, es decir, apto para trabajar con tolerancias muy bajas, gran número de cuchillos y altas velocidades de alimentación. En estas condiciones la herramienta presenta su mejor rendimiento de corte.

Por otra parte, cuando el elemento de corte se deteriora producto de golpear zonas de madera de alta densidad, nudos o elementos extraños, el rectificado permite eliminar tales imperfecciones de la arista de corte, sino esas imperfecciones dejarán trazas sobre la superficie maquinada.

El tamaño mínimo del talón de rectificado alcanza normalmente una magnitud de $0.03 \mathrm{~mm}$, en la práctica siendo imposible lograr valores inferiores, con valores máximos aconsejables a no superar del orden de 0.8 a $1.2 \mathrm{~mm}$. Sin embargo, la práctica común lleva a creer erróneamente que el procedimiento de rectificado aumenta el poder de corte del cuchillo, es decir, mientras más veces se realice, mejor irá siendo la calidad del corte. Siendo por tanto el efecto contrario ya que el aumento del ancho del talón de rectificado tiene por efecto reducir el ángulo de incidencia llevándolo a $0^{\circ} \mathrm{o}$ incluso a valores negativos, lo que implica un efecto del tipo golpeteo de esta nueva (y ancha) arista de corte sobre la nueva superficie a maquinar, con consecuencias negativas en la calidad final del producto (Aguilera 2011).

Los defectos fibra encontrada o grano velloso (fuzzy grain) y fibra astillada o grano arrancado (torn grain) están definidos por la norma ASTM 1666-87 y descritos por Stewart (1980), donde fibra encontrada son fibras o grupos de fibras que no han sido limpiamente cortadas en el maquinado permaneciendo levantadas sobre la superficie. Este defecto está generalmente asociado con madera anormal o fibras gelatinosas que se contraen e hinchan más que la madera normal con los cambios en el contenido de humedad. Bajos ángulos de ataque y cuchillos desgastados son también causantes de este defecto. En cuanto a la fibra astillada, ésta se asocia al corte en sentido contrario a la dirección de la fibra, factores que influyen son un gran ángulo de ataque, profundidad de corte importante, o procesar madera muy seca o muy húmeda.

En relación a otro defecto que también puede estar presente en el proceso de moldurado como es el grano levantado (raised grain), Singh et al. (2010) indica que su causa se debe fundamentalmente a factores inherentes a la materia prima como diferencias de densidad de la madera en la transición madera temprana - madera tardía, al estado del filo de los elementos de corte y al contenido de humedad de la madera, lo cual sumado a lo indicado por otros autores (Hernández y de Moura 2002, Hernández y Rojas 2002) en lo relativo al efecto del desgaste de cuchillos y la reducción del ángulo de incidencia pueden dañar los tejidos de la madera por aplastamiento y compresión.

En relación a los rectificados de la arista de corte, la investigación realizada por Turner (1999) indica que hasta $1 \mathrm{~mm}$ de talón no tiene efecto sobre la cantidad de defectos superficiales en el moldurado de pino radiata, siendo si importantes el ángulo de ataque, el contenido de humedad, el tipo de fibra (fibra corta alrededor de los nudos) y en mayor medida la velocidad de alimentación.

Sin embargo, Hernández y Naderi $(1999,2001)$ al estudiar diferentes niveles de rectificado en varias especies, concluyeron que al aumentarlos progresivamente, esto afecta la superficie y subsuperficie de la madera, comprometiendo el desempeño posterior de las uniones, es decir, las uniones encoladas presentaron un mejor rendimiento cuando el talón de rectificado aumentó (dependiendo de la especie), lo cual fue asociado a una mayor permeabilidad de la madera al haber una mayor rugosidad superficial.

Hernández y de Moura (2002) señalan que al usar cuchillos afilados y sin rectificar se pueden generan superficies con poco daño, sin embargo, al incrementar el área de rectificado del cuchillo y la distancia de material procesado, es posible observar un incremento en el daño a la madera. Por otra parte los autores indican que el desgaste del cuchillo también aumentó cuando se incrementó el área 
de rectificado y la distancia de corte, siendo este proceso similar tanto para la cara de ataque como de incidencia de los cuchillos.

Por otra parte, de Moura y Hernández (2007) indican que la ocurrencia de los defectos de maquinado se incrementa con la profundidad de moldurado y que el efecto de reducción del ángulo de ataque produce un cambio del defecto de fibra astillada hacia fibra encontrada. Asimismo, de Moura y Hernández (2006) y Hernández y Cool (2008) agregan que la ocurrencia de fibra astillada se incrementa al aumentar la velocidad de alimentación en la máquina.

El objetivo de este estudio, es evaluar el efecto del rectificado, cantidad y magnitud, sobre la aparición de defectos fibra encontrada y fibra astillada, en cuchillos de acero rápido (HSS) procesando molduras de pino radiata y asimismo estimar distancia y tiempo de maquinado.

\section{MATERIAL Y MÉTODOS}

Se utilizó madera seca al $12 \%$ de contenido de humedad de pino radiata, estando compuesta la calidad de la madera procesada sobre un $75 \%$ por madera de ancho variable, mientras que la restante corresponde a ancho fijo.

Esta madera fue sometida a un proceso de corte longitudinal para obtener un ancho fijo final de $82 \mathrm{~mm}$, fue trozada donde se liberan defectos como nudos y fue procesada posteriormente en máquina que produce uniones dentadas, para así formar un producto de $4.87 \mathrm{~m}$ de largo, piezas que luego ingresan a máquina moldurera.

Las condiciones de operación de la máquina moldurera fueron las siguientes:

\begin{tabular}{lcl}
\multicolumn{2}{l}{ Velocidad de alimentación : } & $100 \mathrm{~m} / \mathrm{min}$ \\
Velocidad de rotación & $:$ & $6000 \mathrm{rpm}$ \\
Velocidad de corte & $:$ & $60 \mathrm{~m} / \mathrm{s}$ \\
Cabezal de montaje hidro-centrando \\
Diámetro cabezal & $:$ & $191 \mathrm{~mm}$ \\
$\mathrm{~N}^{\circ}$ cuchillos & $:$ & 10 \\
Ángulo de ataque & $:$ & $25^{\circ}$ \\
Ángulo de perfil & $:$ & $52^{\circ}$ \\
Profundidad corte & $:$ & $2 \mathrm{~mm}$ \\
Material de corte & $:$ & acero rápido (HSS) al $12 \%$ de wolframio
\end{tabular}

En estas condiciones se obtuvo una moldura de perfil plano, sobre la cual se evaluaron los defectos de maquinado.

Para poder medir y analizar con mayor exactitud el desgaste del elemento de corte durante el proceso de moldurado, se ocupó una lupa tri-ocular que proporcionó un aumento de 20X. Esta medición se realizó en forma indirecta, es decir, se generó un molde en silicona sobre el cual posteriormente y con ayuda de la lupa fue posible hacer el seguimiento del retroceso del perfil de los cuchillos seleccionados. Los materiales empleados para la formación del molde de silicona, fue una mezcla de silicona (Speedex putty) y un catalizador (Speedex Universal Activator) que acelera el fraguado de la mezcla.

Para obtener un molde que asegure la exactitud de la réplica del cuchillo, se confeccionó un recipiente a base de madera de acuerdo a las dimensiones del cuchillo, de dimensiones internas de $11.5 \times 2.2 \times 2.06 \mathrm{~cm}$. 
El método se dividió en dos partes, en la primera se aplicaron rectificados sucesivos y otra donde sólo se realizó un rectificado inicial.

En el método de rectificados sucesivos, los cuales fueron realizados a criterio del operador según apreciación subjetiva de la calidad, consistió primero en rectificar todos los cuchillos instalados en el cabezal a fin de eliminar tolerancias restantes en la órbita de giro. Posteriormente se procesaron las piezas de madera con las condiciones antes descritas. Con un cronómetro se midió el tiempo entre un rectificado y otro. De esta forma, con el tiempo y la velocidad de alimentación de la moldurera, se calcularon los metros lineales procesados entre cada rectificado.

En el método del rectificado inicial, éste se aplicó para eliminar las tolerancias normales de la órbita de giro, por lo tanto se midieron los defectos generados durante el maquinado bajo estas condiciones, hasta el punto que fuese necesario volver a rectificar dada la aparición de defectos muy graves producto del deterioro de la arista de corte.

En ambos métodos se evaluaron los siguientes aspectos:

- Cantidad total de piezas procesadas en el intervalo

- Número de piezas con defectos

- Tipo y magnitud del defecto (Norma ASTM 1666-87)

- Contenido de humedad en el área próxima del defecto

- Medición de talón de rectificado con molde a base de silicona.

El contenido de humedad se controló según los procedimientos indicados en la Norma Chilena NCh176/1 (INN 1986).

Para evaluar la magnitud del defecto fibra encontrada y fibra astillada, se utilizó como referencia la norma ASTM 1666-87, es decir, siendo los defectos evaluados en este estudio como leve, moderado y grave, haciéndolos equivalentes a los grado 3,4 y 5 respectivamente según la norma indicada. No se consideró en los objetivos de este estudio la evaluación del defecto fibra levantada (grano levantado o raised grain), dándose énfasis sólo a los defectos ya indicados.

El procedimiento que se empleó fue el de aplicar ambos métodos de rectificado sin incluir reafilados intermedios, con lo cual se busca observar la aparición de los defectos mencionados en una situación real de moldurado de pino radiata, es decir, bajo condiciones operacionales corrientemente empleadas en la industria, la única salvedad fue la aplicación de un rectificado único, situación que normalmente no se aplica en estos procesos. Por lo indicado, el efecto desgaste se encuentra presente sobre los resultados, no siendo posible discernir sobre éstos su importancia relativa y la magnitud del rectificado y su consecuencia sobre la generación de los defectos, es decir, se tomará el efecto combinado desgaste - rectificado.

Para poder cumplir de manera confiable el muestreo de piezas en cada uno de los métodos ya mencionados, es necesario determinar un número de piezas que sea representativo a la población total de molduras elaboradas. Para ello se utilizó el siguiente procedimiento:

- Medir las fallas originadas en un número de muestras no menor a 50 unidades en un proceso normal.

- Calcular la varianza de esta muestra y estimar el nivel de confianza y error a utilizar (95\% y 0.01 respectivamente). 
Y la fórmula siguiente:

$$
n_{\infty}=\frac{\operatorname{Var}^{2} * Z_{\frac{\alpha}{2}}^{2}}{E^{2}}
$$

Dónde:

$\mathrm{n}_{\infty}$. Tamaño muestra

Var: Varianza muestra

$Z$ : Nivel de confianza (95\%)

E : error estimado (0.01)

\section{RESULTADOS Y DISCUSIÓN}

Se presentan los resultados para los defectos fibra encontrada (fuzzy grain) y fibra astillada (torn grain) en cada método de rectificado, según intensidad tomando como referencia la norma ASTM 1666-87. $1)$ :

Según el método de rectificado, se consideraron las siguientes condiciones de operación (Tabla

Tabla 1. Descripción de condiciones de operación según tipo de método de rectificado.

\begin{tabular}{|l|c|c|}
\hline Condición operación & Rectificado sucesivo & Rectificado único \\
\hline Piezas procesadas & 10.962 & 6.644 \\
\hline Piezas muestreadas & 1.020 & 500 \\
\hline Metros lineales procesados & 53.460 & 24.300 \\
\hline Tiempo total de maquinado & $680 \mathrm{~min}$ & $300 \mathrm{~min}$ \\
\hline Número de rectificados & 5 & 1 \\
\hline
\end{tabular}

Para el método de rectificados sucesivos, éstos se realizaron de la siguiente manera (Tabla 2):

Tabla 2. Ancho talón rectificado, distancia y tiempo de corte según rectificado.

\begin{tabular}{|l|c|c|c|c|c|}
\cline { 2 - 6 } \multicolumn{1}{c|}{} & \multicolumn{5}{c|}{ Rectificado } \\
\hline Parámetro & 1 & 2 & 3 & 4 & 5 \\
\hline Ancho promedio talón $(\mathrm{mm})$ & 0.33 & 0.75 & 1.27 & 1.31 & 1.44 \\
\hline Distancia $(\mathrm{m})$ & 0 & 810 & 9.720 & 34.020 & 53.460 \\
\hline Tiempo (min) & 0 & 10 & 120 & 420 & 680 \\
\hline
\end{tabular}

En el caso del método de rectificado inicial, éste fue de $0.45 \mathrm{~mm}$, analizándose las piezas hasta el punto en el cual éstas presentaron defectos que hicieron necesario realizar un segundo rectificado, momento en el cual se dio por terminado el muestreo.

Se midió sistemáticamente el contenido de humedad de cada pieza que presentó algún grado de ambos defectos, los resultados indicaron para fibra encontrada una variación entre 9 y $11 \%$, en cambio para fibra astillada el contenido de humedad se situó levemente por bajo el $8 \%$.

Para las condiciones de rectificado sucesivo, el comportamiento de la frecuencia de defectos se presenta en las figuras siguientes: 
En la figura 1 se puede observar un aumento gradual en la frecuencia (porcentaje) de aparición del defecto fibra encontrada en la medida que se incrementa la distancia de maquinado, al mismo tiempo que se incrementa el talón de rectificado, tal como se indica en la tabla 2, a partir del tercer rectificado $(1.27 \mathrm{~mm})$ la frecuencia de defectos se hace mayor. Del total acumulado de defectos, los defectos leves concentraron en 54\%, los moderados el 33\% y los graves el $12 \%$. Es importante agregar que del total de defectos según categoría, tanto los niveles leve y moderado sufrieron un comportamiento homogéneo, sin embargo, el nivel grave de defecto presentó un incremento porcentual superior al observado en los anteriores al superar los 30.000 metros de material procesado. Cabe señalar que en este defecto la madera recién procesada se caracteriza por estar constituida por grupos de fibras de madera que no han sido limpiamente cortadas en la superficie manteniéndose aún levantadas. Esto se puede controlar trabajando con bajos contenidos de humedad y con cuchillos filosos. Como el contenido de humedad de la madera se encuentra entre 9 y $11 \%$, se infiere que la generación de este defecto puede deberse por una pérdida prematura del poder cortante de los cuchillos ya que el avance por cuchillo calculado para las condiciones de operación es de $1.67 \mathrm{~mm}$ lo que equivale a 15 marcas por pulgada, es decir, una muy buena calidad de terminación superficial, y probablemente, además por una calidad regular de los cuchillos (dureza). Debe tenerse en cuenta que el costo de generar una mayor calidad superficial es un mayor desgaste de cuchillos.

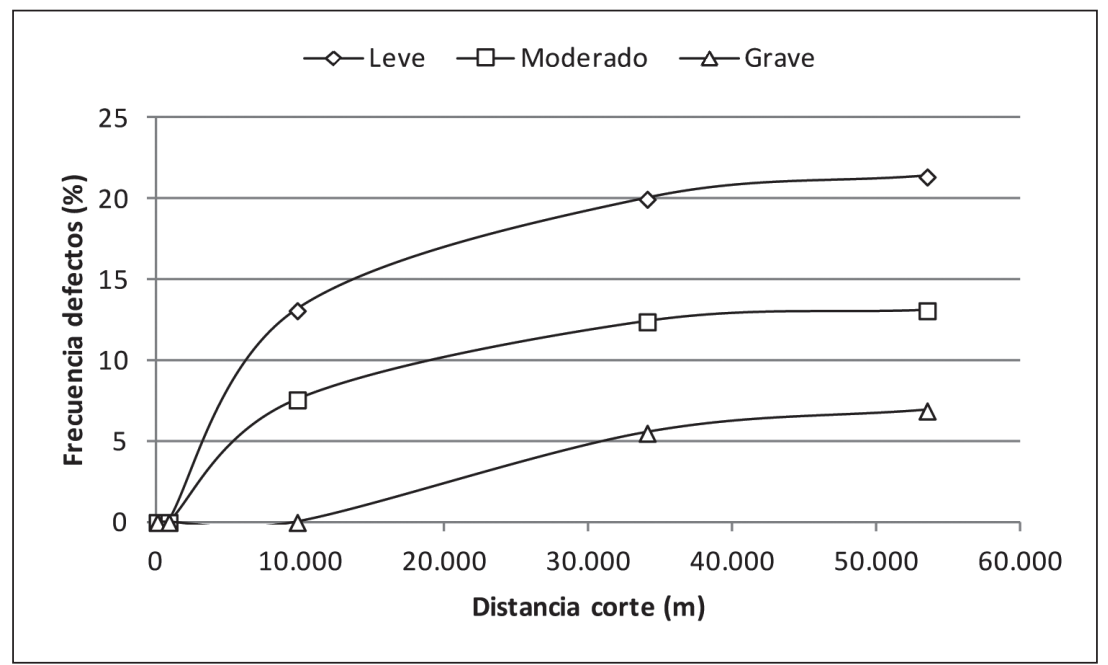

Figura 1. Frecuencia y severidad de defectos para fibra encontrada (fuzzy grain), según distancia de corte en método de rectificado sucesivo

En este caso, hasta el segundo rectificado $(0.75 \mathrm{~mm})$ no hubo ningún efecto sobre la generación de defectos, siendo a partir del tercero, con el incremento gradual del ancho del talón de rectificado $(1.27 \mathrm{~mm})$, pudiéndose inferir esta observación a la presencia de una mayor cantidad de piezas con el defecto de fibra encontrada.

Cuando se realizó un solo rectificado, al inicio del proceso, y luego de una distancia de 24.300 metros de madera procesada, fue necesario corregir la calidad del corte, se puede apreciar en la figura 2 una tendencia similar a los rectificados sucesivos. 


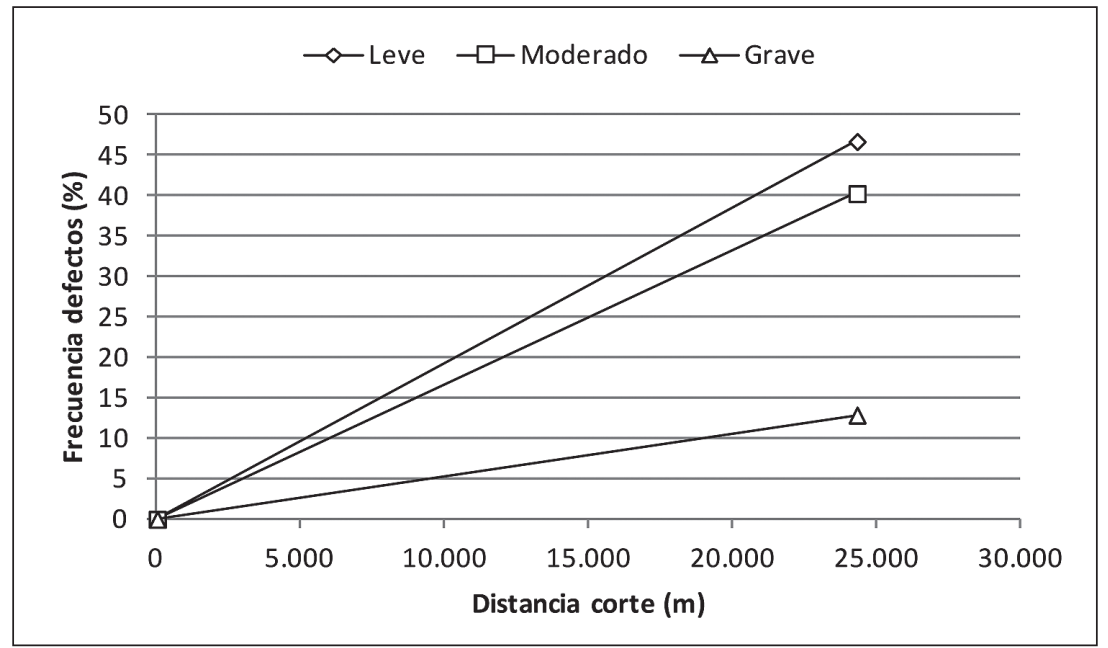

Figura 2. Frecuencia y severidad de defectos para fibra encontrada (fuzzy grain), según distancia de corte en método de rectificado único.

Al confrontar ambos resultados, no queda una evidencia clara de la influencia entre un método y otro sobre la aparición de defectos, lo que se puede inferir es que al aplicar un solo rectificado, es recién al cabo de 24.300 metros procesados (ó 300 minutos de operación) cuando se detectaron los primeros defectos de maquinado, siendo el 13\% de ellos de carácter grave, los que para similar distancia de corte en el método de rectificados sucesivos era inferior al 5\%, además se observó para los otros defectos un $40 \%$ moderado y $47 \%$ leve. Sin embargo, al comparar ambas situaciones (5 versus 1 rectificado), se observó una menor frecuencia acumulada del defecto fibra encontrada cuando se aplicó sólo un rectificado, habiendo por tanto un $27 \%$ más de estos defectos en el método de rectificados sucesivos respecto del rectificado único a una distancia de procesamiento equivalente.

Para el caso de fibra encontrada, no es evidente poder establecer una distancia límite de rectificado, o cual de los dos métodos aplicados es mejor, ya que la aparición de defectos siempre estará presente en este tipo de procesos, siendo incrementada en función de variables inherentes a la materia prima madera, al tipo de acero de los cuchillos, a las condiciones de operación y a la experiencia del operador.

En el caso de fibra astillada (torn grain), este defecto al igual que el anterior mostró un incremento gradual de su frecuencia frente a una mayor distancia de corte y al incremento del talón de rectificado, tal como se puede observar en las figuras 3 y 4 para rectificado sucesivo y único respectivamente.

Además, se puede apreciar una menor frecuencia para los tres niveles (leve, moderado y grave) respecto de fibra encontrada, es decir, en términos porcentuales y para el caso de 5 rectificados, un $68 \%$ de los defectos fueron clasificados como leves, un $29 \%$ moderado y sólo un $2.8 \%$ como graves, en rectificado único un $71 \%$ leve y 29 moderados. Sin embargo, y al igual que el caso del defecto fibra encontrada, para fibra astillada tampoco se hace evidente determinar la distancia límite de rectificado, pero esta vez el rectificado único mostró una frecuencia de aparición de defectos un 35\% inferior respecto del método de varios rectificados. En relación al máximo talón de rectificado factible de generar, los resultados no permiten verificar ese dato ya que para el caso particular del análisis de este tipo de defecto, en general la frecuencia de aparición del defecto fue baja. Cabe señalar que este defecto se caracteriza por una superficie astillada donde pequeñas partículas de madera han sido rotas o levantadas, lo que ocurre cuando los cuchillos están trabajando contra la fibra, a menudo alrededor de nudos o en otros lugares donde se producen cambios bruscos de los ángulos de las fibras. 


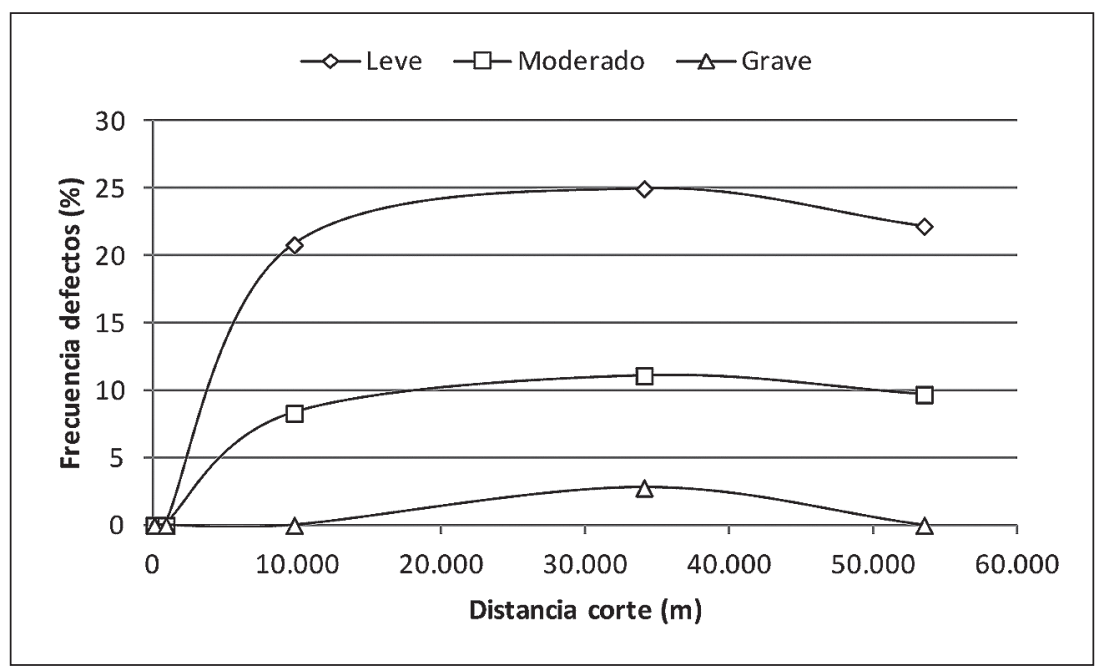

Figura 3. Frecuencia y severidad de defectos para fibra astillada (torn grain), según distancia de corte en método de rectificado sucesivo

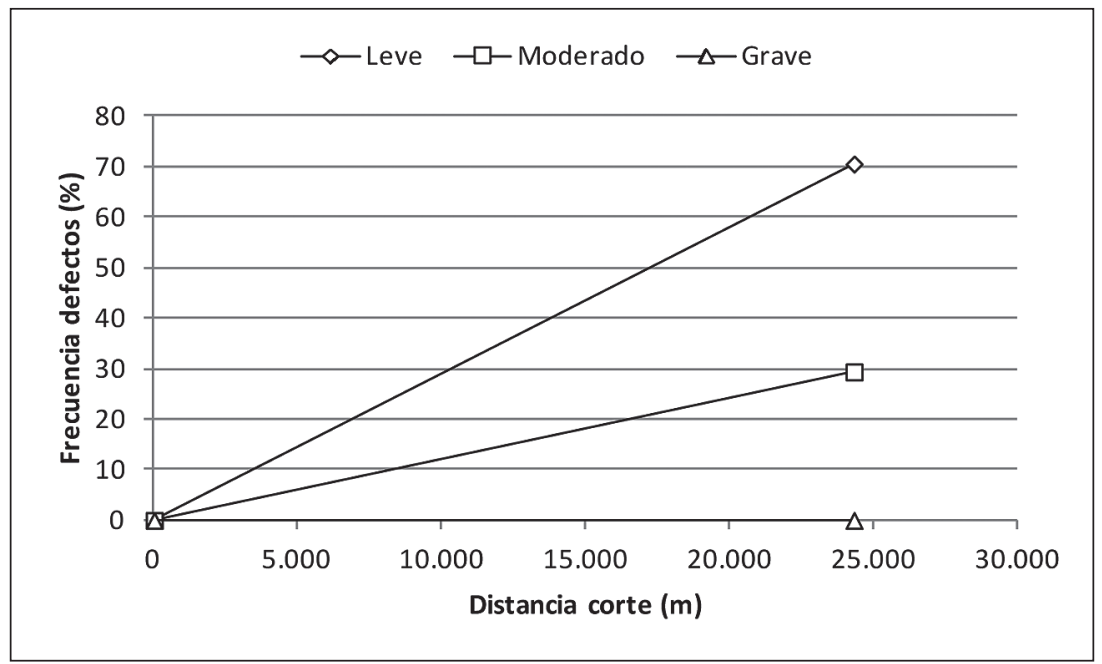

Figura 4. Frecuencia y severidad de defectos para fibra astillada (torn grain), según distancia de corte en método de rectificado único

Al comparar ambas figuras, y cuando se aplica un solo rectificado es cuando se obtiene una frecuencia levemente inferior de defectos respecto del otro método, lo que hace suponer un efecto positivo de minimizar la frecuencia de rectificados. Debe recordarse que las fibras astilladas son un defecto que tiende a disminuir principalmente cuando el filo de la arista de corte presenta un estado óptimo, por ello, una baja calidad de éste obligará a rectificar los cuchillos. El efecto de un solo rectificado implica en este caso un trabajo libre de defectos hasta los 24.000 metros de madera procesada. 


\section{CONCLUSIONES}

El procesamiento industrial de molduras de pino radiata requiere que los cuchillos sean sometidos a un proceso de rectificado inicial con el fin de igualar la proyección de éstos en la órbita de giro y con ello lograr una adecuada calidad de terminación superficial a altas velocidades de giro y de alimentación, este proceso debe ser repetido las veces que sea necesario cuando se detecte alguna irregularidad sobre la superficie de la moldura, sin embargo, el poder cortante no se ve mejorado.

Considerando los resultados obtenidos, se concluye que fibra encontrada se observó con mayor frecuencia al aplicar el método de rectificados sucesivos, estando la composición de estos defectos distribuida en un $54 \%$ en defectos leves, $33 \%$ moderados y $12 \%$ graves, con una frecuencia acumulada $27 \%$ superior de defectos respecto del rectificado único a una distancia de procesamiento equivalente.

En el caso de fibra astillada la secuencia de rectificados sucesivos tienden a generar más defectos respecto del rectificado único, siendo su composición de un $68 \%$ leve, $29 \%$ moderado y $2.8 \%$ leve, y una frecuencia acumulada de defectos superior en un $65 \%$ respecto del rectificado único a una distancia de procesamiento equivalente.

El defecto fibra encontrada se presentó con mayor frecuencia para ambos tipos de método de rectificado en relación a fibra astillada, lo que hace suponer, por la génesis de este defecto, que las fibras al no ser limpiamente cortadas, implica un bajo poder de corte del cuchillo, o mala preparación de éste.

Respecto del defecto fibra astillada, su baja presencia se puede deber a que la materia prima fue adecuadamente trozada, liberando amplias zonas alrededor de los nudos, donde algún remanente de contra fibras fue el que generó la aparición del defecto.

\section{AGRADECIMIENTOS}

Los autores desean agradecer a la Dirección de Investigación y Desarrollo (DID) de la Universidad Austral de Chile.

\section{BIBLIOGRAFÍA}

Aguilera, A. 2011. Monitoring surface quality on molding and sawing processes for solid wood and wood panels. In: Wood machining. Wiley - ISTE Ltd. London, England. pp.159 - 216. ISBN: 978-1-84821-315-9.

ASTM, American Society for Testing and Materials. 2004. Standard Test Methods for Conducting Machining Tests of Wood and Wood-Base Materials. ASTM 1666-87. 19 p.

de Moura, L.F.; Hernández, R.E. 2006. Characteristics of sugar maple wood surfaces produced by helical planing. Wood and Fiber Science 38(1): $166-178$.

de Moura, L.F.; Hernández, R.E. 2007. Characteristics of sugar maple wood surfaces machined with the fixed-oblique knife pressure-bar cutting system. Wood Sci Technol 41(1): 17-29.

Hernández, R.E.; Cool, J. 2008. Effects of cutting parameters on surface quality of paper birchwood machined across the grain with two planing techniques. Holz Roh Werkst 66: 147-154. 
Hernández, R.E.; de Moura, L.F. 2002. Effects of knife jointing and wear on the planed surface quality of northern red oak wood. Wood and Fiber Science 34 (4): 540-552.

Hernández, R.E.; Naderi, N. 1999. Effect of the knife jointing on the gluing properties of wood. Actas del $14^{\text {th }}$ International Wood Machining Seminar, Septiembre 12- 19, Paris, Epinal, Cluny, Francia.: 89-98.

Hernández, R.E.; Naderi, N. 2001. Effect of knife jointing on the gluing properties of wood. Wood and Fiber Science 33(2): 292-301.

Hernández, R.E.; Rojas, G. 2002. Effects of knife jointing and wear on the planed surface quality of sugar maple wood. Wood and Fiber Science 34 (2): 293-305.

INN, Instituto Nacional de Normalización. 1986. Determinación de humedad. Norma chilena NCh 176/1. Of. 1986. Parte 1.

Singh, A.; Dawson, B.; Hands, K.; Ward, J.; Greaves, M.; Turner, J.; Rickard, C. 2010. The anatomy of raised grain on Pinus radiata weatherboards. IAWA Journal 31(1): 67-76.

Stewart, H. 1980. Some surfacing defects and problems related to wood moisture content. Wood and Fiber 12 (3): 175-182.

Turner, J. 1999. Cutting angles and feed speeds for planer-moulding radiata pine. Actas del $14^{\text {th }}$ International Wood Machining Seminar, Paris, Epinal, Cluny, Francia. pp. 53-63. 